\title{
Evaluating cardiac risk: exposure response analysis in early clinical drug development
}

This article was published in the following Dove Press journal:

Drug, Healthcare and Patient Safety

\section{Julie Grenier' \\ Sabina Paglialunga ${ }^{2}$ \\ Bruce H Morimoto² \\ Robert M Lester ${ }^{3}$}

'Data Management and Biometric, Celerion, Montreal, QC, Canada; ${ }^{2}$ Scientific Affairs, Celerion, Lincoln, NE, USA ${ }^{3}$ Global Clinical Research, Celerion, Tempe, AZ, USA
Correspondence: Bruce H Morimoto Scientific Affairs, Celerion, 62I Rose St, Lincoln, NE 68502, US

Tel +l 6502988430

Email bruce.morimoto@celerion.com

\begin{abstract}
The assessment of a drug's cardiac liability has undergone considerable metamorphosis by regulators since International Council for Harmonization of Technical Requirement for Pharmaceuticals for Human Use E14 guideline was introduced in 2005. Drug developers now have a choice in how proarrhythmia risk can be evaluated; the options include a dedicated thorough QT (TQT) study or exposure response (ER) modeling of intensive electrocardiogram (ECG) captured in early clinical development. The alternative approach of ER modeling was incorporated into a guidance document in 2015 as a primary analysis tool which could be utilized in early phase dose escalation studies as an option to perform a dedicated TQT trial. This review will describe the current state of ER modeling of intensive ECG data collected during early clinical drug development; the requirements with regard to the use of a positive control; and address the challenges and opportunities of this alternative approach to assessing QT liability. Keywords: concentration-effect modeling, thorough QT study, intensive ECG collection, intersection union test, QT/QTc, assay sensitivity, positive control, moxifloxacin
\end{abstract}

\section{Introduction}

Prolongation of the cardiac corrected QT (QTc) interval has been a significant focus of the pharmaceutical industry for nearly 30 years. This intense interest first developed in the 1990s when several high-profile, non-cardiac drugs were withdrawn from the US market including the antihistamines terfenadine (withdrawn from the market) and astemizole (withdrawn from the market); the antibiotic grepafloxacin (withdrawn from the market); and the prokinetic medication cisapride (withdrawn from the US market). Reports of sudden cardiac death surfaced once these drugs were introduced to the market and subsequent investigation found that all of these drugs could prolong the QT interval on an electrocardiogram (ECG), particularly in the setting of additional risk factors. The sudden death seen in these cases was attributed to Torsades de Pointes (TdP), a ventricular arrhythmia associated with QT prolongation. This arrhythmia is extremely rare with an estimated incidence of 1 in $100,000,{ }^{1}$ and therefore unlikely to be observed in even large Phase III clinical studies performed during drug development.

Several regulatory guidance documents have been published including the 1997 European Committee for Proprietary Medicinal Products (CPMP) "Points to Consider" which focused on the assessment of QT prolongation by non-cardiovascular drugs. This document was the first formal regulatory guidance on how to address the potential of drugs to cause QT prolongation. Subsequently, the Therapeutic Products Directorate of Health Canada published a preliminary concept paper on the clinical assessment 
of QT prolongation in 2001. Extensive discussion between academia, industry, and regulators resulted in what became the International Council for Harmonization of Technical Requirement for Pharmaceuticals for Human Use (ICH) E14 guideline document titled, "Clinical Evaluation of QT/QTc Interval Prolongation and Proarrhythmic Potential for NonAntiarrhythmic Drugs" which was adopted by the CPMP in 2005. The ICH E14 guideline document introduced the "thorough QT" (TQT) study into the drug development lexicon, and specified that all new chemical entities with systemic bioavailability needed to be clinically tested for the potential to prolong the QT interval, even if preclinical findings did not identify safety concerns. While this regulatory framework has been in place for more than 10 years, reevaluation of the TQT testing paradigm is currently under discussion and development. In this regard, several joint meetings between the US Food and Drug Administration (FDA) and the Cardiac Safety Research Consortium (CSRC) with members from academia, regulatory agencies, and industry have occurred to discuss the future of both preclinical and clinical cardiac safety evaluation in early drug development. ${ }^{2}$ Consensus on preclinical proarrhythmia risk evaluation in conjunction with intensive ECG collection data modeling has helped foster a significant paradigm shift for cardiac safety testing; moving from the TQT study typically done later in drug development to an earlier clinical assessment of potential drug-induced QT prolongation as part of Phase I studies. To this end, Comprehensive in vitro Proarrhythmia Assay initiative, ${ }^{3,4}$ which provides mainly in vitro and in silico data, in conjunction with early phase intensive ECG collection with exposure-response (ER) modeling performed on all available datasets across all drug doses, may help to support the granting of a "TQT waiver" by government regulators. ${ }^{4,5}$ Therefore, while originally treated as a secondary analysis tool, ER modeling of Phase I ECG data is now considered an acceptable alternative to assess proarrhythmic risk in lieu of a conventional TQT study. ${ }^{6-11}$ The present review will describe the current state of ER modeling of intensive ECG data collected during early clinical drug development, and address the challenges and opportunities of this optional approach in evaluating cardiac liability.

\section{What is a conventional TQT?}

A number of excellent review articles have thoroughly discussed the implications of the ICH guidelines and adapted revision for cardiac safety evaluation in a TQT study, ${ }^{6,12,13}$ which will not be discussed here. However, before describing the utility of ER modeling of ECG data, a brief summary of study design elements for a typical TQT study follows. The
ICH E14 guideline indicated that the "threshold of regulatory concern" is a drug-induced effect on the heart rate (HR) QTc beyond an upper bound limit of $10 \mathrm{msec} .{ }^{14}$ A negative TQT study will demonstrate that the one-sided upper 95\% CI for the mean difference in baseline corrected QTc of the drug and the baseline corrected time-matched placebo is below this threshold for all time-points. While several correction factors are available for HR, the guideline specifies that Fridericia's correction factor should be applied in most situations and that Bazett's correction is no longer warranted as it has been shown to be an inferior correction method. ${ }^{10,11}$ Key features of a TQT study typically include exploration of both therapeutic and supratherapeutic drug dose levels, although the guideline only specifies that if not precluded by safety or tolerability concerns, the drug should be tested at substantial multiples of the anticipated maximum therapeutic exposure. The challenge of identifying these doses often pushes the TQT study late into drug development, usually concurrent with Phase II or III clinical trials. Additionally, ICH E14 specifies that a positive control drug, most often the oral $\mathrm{IKr}$ blocking antibiotic moxifloxacin, is required to document assay sensitivity. Therefore, at a minimum, a traditional TQT study will have four treatment arms; placebo, moxifloxacin, and two drug dose levels. With sample sizes varying from 40 to 50 subjects for a crossover design and 100 to 200 and over for a parallel design, a conventional TQT study remains a resource intensive and costly undertaking ranging from 1 to 4 million US dollars. ${ }^{15}$ In light of the high cost of this study design, an effort by all stakeholders is currently underway to develop a more robust preclinical and clinical paradigm with less resource expenditure to profile a drug's cardiac liability rather than conduct a traditional TQT trial.

\section{Applying exposure response modeling to determine proarrhythmia risk}

ER analysis determines the statistical relationship between drug or active metabolite concentrations and clinical data. This type of analysis has been applied to numerous aspects of drug development to describe an acceptable risk/benefit relationship and support regulatory approval, in line with the publication of a guidance document on ER relationship in 2003 by the FDA. ${ }^{16}$ Ever since the adoption of the ICH E14 guideline in 2005, the gold standard for analyzing ECG data from a TQT study has been the intersection unit test (IUT). This binary analysis basically consists of a by-time-point comparison between a drug and placebo of the time-matched baseline-adjusted QTc intervals. If the upper bound of the 
1 -sided $95 \% \mathrm{CI}$ around this difference is above $10 \mathrm{msec}$ for at least one time-point, the study is declared positive.

In 1976, the effect of the antiarrhythmic agent procainamide on the QT interval was the first application of cardiodynamic ER modeling where hysteresis was observed when plasma concentrations of procainamide were plotted against changes in QTc. ${ }^{17,18}$ Multiple efforts have been made in the last decade to establish the utility of ER linear or nonlinear mixed-effects models to assess potential drug-induced QT/ QTc prolongation in a less dichotomous fashion than the by-time-point analysis. As a first step in the comparison of the two approaches, the ER models were investigated by analyzing data from dedicated TQT studies while evaluating the performance of alternative analyses to the IUT. ${ }^{19-25}$ Garnett et $\mathrm{al}^{26}$ also described several case studies where ER modeling of TQT results played a pivotal role in data interpretation: to identify risk of QTc prolongation at lower doses than those studied in a TQT; to predict proarrhythmia risk in an underpowered TQT study when data were pooled from previous studies; and to evaluate assay sensitivity of a positive control. Moreover, it has been suggested to apply ER analysis to TQT data as a complementary method for assessing proarrhythmia risk when a drug is suspected of inducing QTc prolongation. ${ }^{27}$ As a secondary analysis, ER analysis has assisted with compound selection for further development, guided dose selection, and TQT study design, and even resulted in program discontinuation if an unfavorable QT risk was identified in early development. ${ }^{26,27}$ Finally, to further support this new testing paradigm, ER modeling was prospectively evaluated in a recent single ascending dose (SAD) type study. In a collaborative effort, the Consortium for Innovation and Quality in Pharmaceutical Development and the CSRC (IQ-CSRC) examined known marketed drugs that induce QTc prolongation above the threshold of regulatory concern (ondansetron, quinine, dolasetron, moxifloxacin, and dofetilide). The results from this investigation confirmed the known QT liability of the respective compounds and provided evidence that intensive QT assessment and modeling in early phase clinical development is an appropriate alternative to a traditional TQT trial. ${ }^{28}$ The ER approach has now become accepted as a primary analysis tool, as acknowledged in the ICH E14 Q\&A R3 document first issued in December 2015 ${ }^{10}$ and reissued in FDA ICH E14 R3 guidance in June 2017. ${ }^{11}$

Modeling and simulation of pharmacokinetic and pharmacodynamic (PK-PD) data in a Bayesian framework have been used for decision making in many other areas of drug development to try to explain the structure and relationship between PK, PD, and safety data in a comprehensive manner.
QT prolongation as a PD marker has taken a longer route into the realm of modeling and simulation. This delay in approach can partly be explained by the fact that the assessment of QT prolongation has been based on dedicated studies designed for inferential statistics, ${ }^{29}$ whereas other biomarkers are more often measured throughout multiple clinical studies and are part of multiple varying endpoints. In a recent review, France and Della Paqua ${ }^{29}$ described the advantages and disadvantages of population PK-PD modeling. In general, population ER models are much more flexible and can account for hysteresis, time-dependent effects, covariates such as demographics, linear and non-linear relationships, and can more easily merge data from multiple studies. However, one can easily imagine the number of models that can emerge from these analyses. From a regulatory perspective, it may be cumbersome to review different sets of models for each submission, and it may be more desirable to maintain a balance between trying to find the best model to explain the data and trying to determine the minimal model that would be sufficiently informative to give an answer to the question: "Is there drug-induced QT/QTc prolongation?"

\section{Key features of a linear model}

Efforts have been put forth to develop a pre-specified model in order to standardize data analysis and facilitate regulatory agencies' review. Another advantage of pre-specifying a base model with few variations for ER analysis is that it may save time by not trying to fit an endless number of models. Huh and Hutmacher ${ }^{30}$ have looked at the performance of three different linear mixed-effects models using simulated PK and PD data. Interestingly, the simpler model performed well by showing minimal bias on the final estimate of QTc prolongation and had the non-negligible advantage of not having convergence issues that would limit its use. Conrado et $\mathrm{al}^{9}$ have performed a meta-analysis of ECG data from 39 SAD or multiple-ascending dose (MAD) studies comparing different models. Among other factors, five unique baseline models were tested and it appears from these results, that an unstructured baseline model (i.e., with a baseline value for each time-point) was selected most often for QT/QTc. This type of meta-analysis is of great interest for defining pre-specified models. One must not forget that good science should always drive the analyses and that one size may not fit all. However, it is scientifically sound to at least start with a model that has a better likelihood of fitting the data, keeping in mind that every model has assumptions and that these assumptions have to be fulfilled for the model to be valid. Although there is no official consensus on a specific linear 
model, one can nonetheless make a very general statement of a linear model, describing the change from baseline QTcF $(\triangle \mathrm{QTcF})$ for a parallel design could be as follows for subject $\mathrm{i}$ on treatment $\mathrm{j}$ at time $\mathrm{k}$ :

$$
\begin{gathered}
\Delta \mathrm{QTcF}_{\mathrm{i}, \mathrm{i}, \mathrm{k}}=\left(\text { treatment }_{\mathrm{j}}+\text { time }_{\mathrm{k}}\right)+\text { Baseline }_{\mathrm{i}}+ \\
\text { Slope }_{\mathrm{i}, \mathrm{j}} \times \text { Concentration }_{\mathrm{i}, \mathrm{j}, \mathrm{k}}+\varepsilon_{\mathrm{i}, \mathrm{j}, \mathrm{k}}
\end{gathered}
$$

where $\varepsilon$ is the residual variability. The $\Delta$ QTcF from subjects on placebo and active drug are pooled for the analysis and the placebo-corrected change from baseline QTcF $(\Delta \Delta \mathrm{QTcF})$ can then be computed using the geometric mean Cmax observed in different scenarios of clinical interest, such as hepatic or renal impairment, food effect, or drug-drug interaction, which are usually used to determine the worst case clinical scenario. Of note, diurnal variation of the QT/QTc intervals is accounted for by the time-matched placebo data. As a result, pre-dose ECG data are sufficient for baseline determinations and there is no need for a full day of time-matched ECG collection prior to dosing. This is of great interest too when trying to reduce the cost of cardiovascular risk assessment.

The first assumption that must be verified before starting the analysis of QT data is the appropriateness of the HR correction factor for the QT interval. The most common HR correction factor for $\mathrm{QT}$ is Fridericia $\left(\mathrm{QTcF}=\mathrm{QT} / \mathrm{RR}^{1 / 3}\right)$. However, in the presence drug-induced HR changes, other correction methods need to be explored. Individual correction or population based correction methods using linear mixedeffects models are available. ${ }^{31}$ Moreover, the individual correction method requires obtaining a wide range of $\mathrm{HRs}$ from the ECG collection and it is usually assessed by collecting ECG data over a 24-hour period prior to dosing. It is also worth mentioning that regulatory documents do not mention a specific point where a change in HR would be considered significant, although Hossain et $\mathrm{al}^{32}$ have used a threshold of $8 \mathrm{bpm}$ in their analysis of the effect of the antibacterial drug gepotidacin on the QT interval.

The second assumption for using a linear mixed-effects model in the context of potential drug-induced QT/QTc prolongation is the absence of hysteresis. The collection of both ECGs and PK samples is time-matched and modeling these data simultaneously assumes a direct effect, if any exists. Beyond the visual inspection of superimposed concentration and QT/QTc versus time profiles, ${ }^{5}$ the absence or presence of hysteresis may be assessed statistically. When $\Delta \Delta \mathrm{QTC}$ exceeds $5 \mathrm{msec}$ for at least three time-points, the time to peak for both concentrations and $\Delta \Delta \mathrm{QT}$, can be compared using a one-sided one-sample Wilcoxon test with an $\alpha$ of 0.01 . A difference exceeding 1 hour would suggest the presence of hysteresis and therefore the linear direct model would most likely be inappropriate. ${ }^{28}$ Another method to assess hysteresis using the derivative of drug concentration versus time and standardized residuals from the fitted model has been reported. ${ }^{9}$ This prior assessment may avoid the need for testing models with delayed effects, keeping in mind that reducing the number of models tested reduces both analysis time and review time.

The third major assumption is linearity itself. Although the intercept term (including for example, treatment and time) may allow room for some model misspecification, the appropriateness of a linear model has to be verified when there is the appearance of a potential drug-induced effect on the QT/QTc interval. Standard diagnostic plots such as QQ plots and standardized residuals are to be looked at carefully. The significance of a quadratic term may also be tested, and when this term is significant, nonlinear models may be tested (Emax, sigmoidal, and log-linear). ${ }^{28}$ Again, this is in the context of trying to limit the number of models to be tested by assuming linearity from the start in order to streamline the analysis and review process, and have the minimal parametrization to answer the primary question about the presence or absence of drug-induced QT/ QTc prolongation. However, in the more general context of modeling and simulation, the type of relationship between a response and the concentration is not necessarily assumed and both linear and nonlinear models are usually tested from the start to see which best describes the relationship. There are numerous examples in the literature of more elaborate modeling strategies of QT data. ${ }^{9,21,24,25,33}$

\section{ER analysis and assay sensitivity}

The ability to reproducibly detect small changes in QT/QTc prolongation in a given setting is crucial. In TQT studies, assay sensitivity is established with the use of moxifloxacin using a by-time-point analysis, where the lower limit of the 2-sided $90 \% \mathrm{CI}$ around the mean $\Delta \Delta \mathrm{QTc}$ has to be above $5 \mathrm{msec}$ for at least one time-point. On average, a $400 \mathrm{mg}$ oral dose of moxifloxacin induces a mean effect of $\sim 8-15$ msec 2-4 hours following dosing. ${ }^{13,20}$ One would expect to be able to show the same effect magnitude using ER analysis. The characterization of the QT/QTc prolongation versus moxifloxacin concentration has been performed using complex models and different sources of data (TQT, $\mathrm{SAD}$, pooled data across different studies, and data from a single study) consisting of various sample sizes. All have shown that the expected magnitude of prolongation seen in TQT studies could be obtained through ER modeling, 
therefore demonstrating assay sensitivity. Using pooled 400 mg oral dosing of moxifloxacin and placebo data from four TQT studies ( $\mathrm{n}=99)$, Grosjeanand and $\mathrm{Urien}^{21}$ developed a population PK-PD model using nonlinear mixed-effects analysis. The input concentrations used in the PK-PD model were the fitted concentrations from a previously established population PK model on the same set of data. Briefly, the strategy used for this analysis was to incorporate the QTc interval of placebo as the dependent variable and have the correction for HR as part of the model, along with a term accounting for circadian rhythm using cosine functions (oscillators with various amplitudes and frequencies). The effect of moxifloxacin on the QTc was then evaluated using a variety of linear, Emax, and sigmoidal models. An additive Emax model was selected for best fit ( $\sim$ Emax $\times$ concentration/ [EC50+concentration]). This same approach was used to evaluate the moxifloxacin-induced QTc prolongation in a study of Korean male subjects. ${ }^{33}$ An Emax model was also selected but different parameter estimates were obtained, mostly because moxifloxacin data also included an $800 \mathrm{mg}$ dose in addition to $400 \mathrm{mg}$ ( $\mathrm{n}=27$ for each dose and placebo, in a crossover study design). These very detailed models delineated the different sources of variability and not only described the drug-induced prolongation but also the placebo, baseline, and diurnal effects. However, whether or not such complex models are needed for testing assay sensitivity of a study is an important unanswered question. Other simpler linear models have been published for moxifloxacin. Shah et $\mathrm{al}^{22}$ tested the ER linear model using data from a TQT study $(n=25$ males, 56 total) and data from an SAD study $(n=24$ males). In another investigation, moxifloxacin-induced QTc prolongation was compared between Caucasian and Japanese healthy subjects, using ER analysis with a single dose of $400 \mathrm{mg}$ and comprising 40 subjects in each group. ${ }^{34}$ All the aforementioned ER analyses with moxifloxacin have however used substantial sample sizes that are not representative of the number of subjects typically enrolled in an SAD study. Panicker et $\mathrm{al}^{20}$ examined data from a TQT study in order to build 1000 datasets with 8:8 moxifloxacin:placebo subjects in a parallel design by resampling with replacement for each group. Each of the 1000 datasets was analyzed with an ER linear model and the model based $\Delta \Delta \mathrm{QTc}$ at the geometric mean Cmax of moxifloxacin was estimated with its $90 \% \mathrm{CI}$. The results from these analyses indicated a statistical power of $89 \%$ to show an effect (i.e., upper bound of the $90 \% \mathrm{CI}$ above $10 \mathrm{msec}$ ) with a small sample size. Furthermore, moxifloxacin was one of the drugs examined in a prospective study employing two dose levels (400 mg orally and $800 \mathrm{mg}$ via a 60-minute intravenous infusion). Nine subjects received moxifloxacin and six received placebo. The authors reported that nonlinearity was not significant for moxifloxacin. ${ }^{35}$ Furthermore, the potential delay in the effect of moxifloxacin on the QT/QTc interval was explored with other factors in a meta-analysis pooling the results from 20 TQT studies. The estimated 30-minute lag time in reaching equilibrium is still within the 1-hour range for declaring no hysteresis. ${ }^{25}$ Considering the available literature on the modeling of QT/QTc versus moxifloxacin concentrations, it suggests that a linear model may not necessarily be the best model but can still be sufficient for the purpose of determining assay sensitivity using a small number of subjects, as would be expected from an SAD-type study design.

\section{Minimizing false-positive and false- negative rates}

Modeling and simulation of data from a standard TQT study with moxifloxacin and placebo treated subjects, concluded that 8-9 subjects on active drug are sufficient to detect a drug-induced QTc prolongation with an estimated power greater than $80 \%{ }^{36}$ This sample size is typical for an SAD study with 6-9 active subjects and 2-3 placebo controls per dose. Pooling placebo controls across investigated doses to achieve a minimum of six subjects is sufficient for ER modeling of the data. ${ }^{36}$ Moreover, sub-analysis from five TQT studies with a sample size of nine on active drug and six on placebo modeled a false-negative rate of $5 \%$ or lower from moxifloxacin exposure and other drugs with a large QTc effect. ${ }^{19}$ However, it is unlikely that all SAD-type studies with extensive ECG collection would include subjects receiving moxifloxacin, especially for first-in-human (FIH) SAD studies, therefore, other methods for assessing assay sensitivity must be considered if the range of drug exposure does not give sufficient confidence with regard to false-negative results. Few methods have been proposed in the literature, mostly based on Monte Carlo simulations of thousands of studies from which the frequency of a specific outcome is assessed. Nelson et $\mathrm{al}^{5}$ used their selected model from the $\triangle$ QTcF versus GS4997/metabolite A analysis during their FIH study to assess false-negative and false-positive rates under specific conditions. To test for the false-negative rate, they imputed a slope so that there was a $10 \mathrm{msec}$ prolongation at supratherapeutic concentrations. Results from 1000 simulated studies showed a false-negative rate of $6.5 \%$. In the same manner, 1000 studies were simulated under the assumption of a true effect of $3 \mathrm{msec}$ at the supratherapeutic dose. A $19.1 \%$ false-positive rate was obtained from those 
simulations. In comparison, TQT studies have typically been conducted using at least 40 subjects per study arm in order to achieve $80 \%$ power.

ER modeling of QT data has served to reanalyze the data obtained in a TQT study and confirm the original conclusions. ${ }^{24}$ ER analysis has also been useful to help clarify the interpretation of ambiguous results from TQT studies as shown by the following example. The primary statistical analysis of a TQT study performed for losmapimod (GlaxoSmithKline), a selective $\mathrm{p} 38 \alpha / \beta$ MAPK inhibitor, not only had failed to demonstrate a lack of effect but it also appeared, from the by time-point analysis, using the IUT, that the therapeutic dosing regimen (7.5 mg BID) triggered a larger QT prolongation than the supratherapeutic dosing regimen (20 mg QD). ${ }^{23}$ Reanalysis of the data using a concentration QT approach gave another perspective on the data, and suggested that the inconclusive results of the by-time-point analysis could be due to a shift in baseline after multiple dosing, or a baseline difference between treatments. Results from the ER analysis thereby suggested that the original results from the IUT were likely to be false-positive.

\section{Positive control requirements}

In the context of an ER analysis during an intensive ECG collection study, a positive control is not necessarily required if multiples of exposure are evaluated. If the range of doses cannot be safely met in an SAD/MAD Phase I study, then the use of a positive control will likely be required. Although adding a moxifloxacin arm to an SAD-like study is preferred, ${ }^{22}$ there are other approaches that have been advocated to confirm assay sensitivity, including the effects of feeding subjects a meal as promulgated by Taubel et al. ${ }^{37-39}$ In an early study, a meal was used to evaluate assay sensitivity during an SAD study with intensive ECG collection investigating the effect of E-52862, an analgesic sigma-1 receptor antagonist, on proarrhythmia risk. This study demonstrated promising and consistent results as evidenced by shortening of the QTc interval by $-8.1(90 \% \mathrm{CI}:-10.4,-5.9) 1$ hour and $-7.2(90 \%$ CI: -9.4, -5.0) 3 hours after meal consumption. ${ }^{40}$ Additionally, in a more recent review, computer simulation was applied to assess the effects of a meal on the QTc interval applied to three different crossover study designs with varying numbers of subjects. The authors documented that food can reliably show assay sensitivity for small cohorts consisting of at least 18 subjects with a power greater than $80 \%$, although they recognized that this approach may need to be tested in studies involving larger numbers of individuals or different study designs. ${ }^{39}$ In addition, the QT effects of postural change to demonstrate assay sensitivity have been suggested by Wheeler, ${ }^{41}$ although this physiologic approach has not gained any traction within the industry. While the aforementioned interventions may eventually find their way into regulatory guidance, they are not yet required and additional effort to reproduce and validate these non-pharmacologic assays across a broad range of drugs and study designs is ongoing. ${ }^{25}$

\section{Lessons learned and points to consider}

As the role for concentration effect modeling has now come to the forefront, the basis for declaring this as a primary analysis tool is of interest and multifactorial. These factors include good concordance with the IUT, multiple simulation studies done both by regulators and academics supporting its value, the knowledge gained over 10 years of employing this type of analysis in hundreds of QT trials, and the findings of the IQ-CSRC study documenting the utility of this approach with a small group of subjects in an SAD-like protocol design. While economic factors may govern the decision whether to incorporate ER analysis in an FIH protocol, there are less expensive options that could also be considered. One of these would entail collecting the ECG data but not analyzing it until later phase/proof of concept studies are completed, since the majority of drugs under development will not proceed beyond Phase II. Another option would be to simply analyze the data in the highest cohorts of the SAD study rather than in the lowest dosed cohorts. This of course assumes that the highest dose levels planned in the study design will be achieved prior to reaching a maximum tolerated dose, so as to have a robust dataset available for analysis.

There are a number of critical elements to be considered when employing ER analysis as a primary tool in support of a TQT waiver. These factors involve ensuring that adequate drug exposures are achieved for both the parent moiety and any metabolites after single doses, or at steady state upon multiple dosing for agents that are affected by intrinsic and extrinsic factors, such as the effect of food, drug-drug interactions, altered metabolism, age, gender, and hepatic or renal impairment. Also pivotal is the understanding that the Cmax of a compound is not equivalent to the highest clinically relevant exposure, and that the highest clinically relevant exposure is not the same as the supratherapeutic drug dose. Finally, the supratherapeutic dose that would be targeted in a TQT trial will often be less than the FDA-proposed mandate ${ }^{3}$ that the investigational drug should achieve an exposure of at least two times the "highest clinically relevant exposure" replicating a worst case clinical scenario. As such, the 
margin for patient safety and the likelihood of false-negative responses are optimally addressed by analyzing data surrounding the Cmax, the highest clinical exposure and at the time-point when a multiple of the highest relevant clinical exposures occur.

The original ICH E14 document ${ }^{14}$ stated that "the confidence in the ability of the study to detect QT/QTc prolongation can be greatly enhanced by the use of a concurrent positive control group (pharmacological or non-pharmacological) to establish assay sensitivity". A key message from the most recent FDA guidance ${ }^{11,42}$ pertaining to concentration effect modeling is that a positive control is not necessary to establish assay sensitivity if the collected information covers a sufficient range of exposures, and the study is conducted in a controlled environment so as to produce high quality ECGs. However, there is still uncertainty surrounding the optimal approach to document assay sensitivity when adequate exposures are not achieved. Adding a sufficient number of placebo subjects to each dosing cohort or running a separate placebo cohort has been suggested, although the proper number of placebo subjects will vary depending on the trial design and the compound under investigation. Alternatively, there are several potential non-pharmacologic strategies (as described previously) to establish assay sensitivity in this setting so as to control for diurnal variation, limit bias, and reduce the likelihood of a false-negative study. Chief among these is the publication by Ferber et $\mathrm{al}^{43}$ concerning method bias sensitivity which has yet to be validated. In this study the slope of Bland Altman plots was utilized as a bias metric. They compared QT measurements using their core laboratory algorithm with a fully automated computer program, and benchmarked these findings against the five drugs studied in the IQ-CSRC trial which had known cardiac safety signals. When bias severity was greater than $-20 \mathrm{msec}$ over a range of QTc values of $100 \mathrm{msec}$ or greater, the ability to predict the QT effects of the five studied drugs failed. Contrariwise, when there was a QT difference of $<-10 \mathrm{msec}$ bias severity, then the chance of a false-negative finding was reduced to less than $5 \%$. They concluded that a metric of bias severity "has to be included" in all reported QTc information obtained in early phase studies using exposure response analysis to mitigate against false-negative results. However, this type of methodology implies arbitrary thresholds and is of a more exploratory nature as the sensitivity and specificity are difficult to estimate.

There are circumstances where the use of concentration effect modeling can be quite challenging, and not all drugs are candidates for this analysis. One of these is with drugs that demonstrate significant HR variability such that an alternative to the population based QTcF correction factor would need to be utilized. The magnitude of HR change that would require a different correction factor to optimally regress QT against HR is not well delineated, nor is the number of QT-RR observations necessary to perform a proper regression established. Couderc et al ${ }^{44}$ suggested 400 QT-RR pairs, an HR range between 60 and $100 \mathrm{bpm}$, and T wave amplitude of $3 \mathrm{mV}$ would be optimal to derive an individual correction factor when the population based formulae are not adequate. A recent FDA symposium ${ }^{42}$ recommended a full pre-dose day of monitoring to properly compute an individual baseline correction of the QT, while others have proposed shorter pre-dose monitoring periods. A second potential limitation of modeling is drugs in which both the parent and metabolite may inhibit the hERG channel, and their effects on the QTc interval may not be appropriately captured in a single dose escalation study, if the parent and metabolite do not accumulate at the same rate after multiple dosing. A third class of drugs which can create insufficient modeling information is those entities which are time released, whereby the model may only profile a narrow concentration range of the test compound. Lastly, as mentioned earlier in this review, drugs with delayed effects and hysteresis require careful timing of ECG and PK samples and evaluation of models accounting for the delayed effect.

Between December 2015 and December 2016, there were 25 exposure response proposals submitted to the QT-IRT of the FDA for review, of which 11 were approved and 14 declined..$^{45}$ The regulators' basis for declining these proposals was that they did not satisfactorily characterize the drug's liability either due to an inadequate prespecified modeling, design, and analysis plan, or that the protocol failed to document an adequate exposure range of the investigational agent. Other shortcomings in these proposals included failure to routinely sample out to 24 hours or at the Cmax when an active metabolite was present, failure to consider the effects of meals, failure to consider the baseline QTc as a covariate for the QTc model, and inappropriate pooling of studies that were collected in a heterogeneous fashion. Lastly, there are other elements of an exposure response proposal that are an integral part of a regulatory submission, and might prompt rejection of the proposal if they are omitted. These entail exploratory plots to test model assumptions, a linear mixedeffects model or other alternative model such as Emax, and comprehensive treatment-placebo difference calculations.

In light of the relatively high rate of rejected submissions noted and to delineate the critical elements of ER analysis necessary for regulatory submission, stakeholders 
are currently drafting a best practice document conforming to good practice guidelines. ${ }^{46}$ This analysis plays a pivotal role when deployed in FIH single dose escalation studies intended to support a TQT waiver. While SAD study designs are the preferred strategy for incorporating this analysis, ER modeling in an MAD study would be indicated when there is drug accumulation, active metabolites or drugs with a long half-life. Moreover, data from multiple studies may also be combined to support a TQT waiver if they are conducted in a homogeneous manner with consistent clinical conduct and robust ECG acquisition methodology and measurement, thereby minimizing any variability. In addition to its role in advocating for a TQT waiver, performing exposure response QTc analysis offers the advantages of clarifying ambiguous results from use of the IUT, enabling data to be generated in challenging populations such as oncology patients, and facilitating extrapolation of a compound's cardiac liability at doses that may not have been studied but are clinically relevant.

\section{Conclusion}

It is evident that ER analysis has become a highly valued and integral part of assessing a drug's QT liability, and will increasingly be incorporated into drug development programs in lieu of performing a dedicated TQT study. However, the utility and focus on the QT interval as a surrogate marker for ventricular arrhythmias and TdP, although deeply entrenched, have been in question since ICH E14 ${ }^{14}$ and preclinical guideline (such as S7B) ${ }^{47}$ documents were initially drafted. One issue is that the threshold of regulatory concern of $10 \mathrm{msec}$ QTc prolongation for small molecules is quite conservative, and has likely excluded drugs from development that may have been beneficial. A second issue is that the positive predictive value of QT prolongation for arrhythmias is relatively modest, and the QT interval is not highly specific for identifying a drug's cardiac liability. This is evidenced by multiple agents which may cause QT lengthening and are not proarrhythmic, especially those demonstrating multichannel ion block. Similarly, the drug arsenic trioxide, despite profound QT prolongation, was approved for use in the treatment of acute myelocytic leukemia, thereby underscoring that regulators must routinely balance a drug's benefit against risk especially in the realms of rare diseases and oncologic agents. Finally, it is problematic that in clinical practice, many practitioners may not be aware of a drug's black box warning and potential for QT prolongation. Accordingly, the mission of protecting the public domain, while embraced by regulators, is a shared responsibility and better education of health care providers and additional safeguards need to be instituted to ensure patient safety when drugs that affect cardiac repolarization are prescribed.

It is important to recognize that there are additional ECG biomarkers of interest, which also merit investigation and may play an increasingly important role when combined with ER analysis. As an example, post hoc assessment of T wave morphology (asymmetry, double peaking, and flatness) in conjunction with QT interval measurements may help identify individuals who have a higher proarrhythmic risk. ${ }^{48}$ Similarly, we have learned that some drugs, such as Amiodarone and Verapamil, demonstrate multichannel ion channel block and while they lengthen the QT interval, they are actually antiarrhythmic and not proarrhythmic due to either late $\mathrm{Na}^{+}$or L-type calcium current block. ${ }^{49}$ In this regard, in subjects who manifest QT drug prolongation during early phase development, measuring the end of the QRS complex to the end of the T wave corrected for HR (the J-Tc interval) and separating the $\mathrm{J}-\mathrm{Tc}$ interval into $\mathrm{J}-\mathrm{T}_{\text {peakC }}$ and $\mathrm{T}_{\text {peakC }}-\mathrm{T}_{\text {endC }}$ components, may prove to risk stratify these individuals into low and high risk proarrhythmic pools. To address this more directly, there is now FDA-released open source software which enables measurement of these intervals and these data may become a required ECG biomarker for new drug submission. ${ }^{50}$

Lastly, it is good to keep in mind that the evaluation of a drug's proarrhythmic liability goes beyond simple measurement of the QTc interval vis-a-vis a traditional TQT trial or by ER analysis in an FIH study. To this point, there may be offtarget effects involving the cardiovascular system that should be addressed during development. These can encompass effects on cardiac muscle and valves, the coronary arteries, pulmonary and systemic arterial vasculature, and thrombogenicity, all of which can be evaluated by selecting from a comprehensive suite of non-invasive imaging and serologic diagnostic tests.

\section{Disclosure}

JG, SP, BHM, and RML are employees of Celerion. The authors report no other conflicts of interest in this work.

\section{References}

1. Sauer AJ, Newton-Cheh C. Clinical and genetic determinants of torsade de pointes risk. Circulation. 2012;125(13):1684-1694.

2. Gintant G, Sager PT, Stockbridge N. Evolution of strategies to improve preclinical cardiac safety testing. Nat Rev Drug Discov. 2016;15(7):457-471

3. cardiac-safety.org [homepage on the Internet]. Critical DiscussionFuture of the Assessment of Drug-Induced Arrhythmias and the Comprehensive in Vitro Proarrhythmia Assay (CiPA). Cardiac Safety Research Consortium; 2016. Available from: http://cardiac-safety. org/2016/12/critical-discussion-future-of-the-assessment-of-druginduced-arrhythmias-and-the-comprehensive-in-vitro-proarrhythmiaassay-cipa/. Accessed March 8, 2018. 
4. Fermini B, Hancox JC, Abi-Gerges N, et al. A new perspective in the field of cardiac safety testing through the comprehensive in vitro proarrhythmia assay paradigm. J Biomol Screen. 2016;21(1):1-11.

5. Nelson CH, Wang L, Fang L, et al. A quantitative framework to evaluate proarrhythmic risk in a first-in-human study to support waiver of a thorough QT study. Clin Pharmacol Ther. 2015;98(6):630-638.

6. Darpo B, Nebout T, Sager PT. Clinical evaluation of QT/QTc prolongation and proarrhythmic potential for nonantiarrhythmic drugs: the International Conference on Harmonization of Technical Requirements for Registration of Pharmaceuticals for Human Use E14 guideline. $J$ Clin Pharmacol. 2006;46(5):498-507.

7. Piccini JP, Whellan DJ, Berridge BR, et al. Current challenges in the evaluation of cardiac safety during drug development: translational medicine meets the Critical Path Initiative. Am Heart J. 2009;158(3):317-326.

8. Cavero I, Holzgrefe H, Clements M. The prospective IQ-CSRC trial: a prototype early clinical proarrhythmia assessment investigation for replacing the ICH E14 thorough QTc (TQT) study. J Pharmacol Toxicol Methods. 2016;80:1-8.

9. Conrado DJ, Chen D, Denney WS. Cardiovascular safety assessment in early-phase clinical studies: a meta-analytical comparison of exposure-response models. CPT Pharmacometrics Syst Pharmacol. 2016;5(6):324-335.

10. International Conference on Harmonisation of Technical Requirements for Registration of Pharmaceuticals for Human Use. Implementation Working Group ICH E14 Guideline. The clinical evaluation of QT/QTc interval prolongation and proarrhythmic potential for non-antiarrhythmic drugs. $Q \& A$ R3. International Conference on Harmonisation of Technical Requirements for Registration of Pharmaceuticals for Human Use; 2015. Available from: http://www.ich.org/fileadmin/Public_Web_ Site/ICH_Products/Guidelines/Efficacy/E14/E14_Q_As_R3_Step4. pdf. Accessed March 8, 2018.

11. US Food and Drug Administration. FDA Guidance for Industry E14 (2017) Clinical Evaluation of QT/QTc Interval Prolongation for NonAntiarrhythmic Drugs - Questions and Answers (R3). Food and Drug Administration; 2017. Available from: https://www.fda.gov/ucm/groups/ fdagov-public/@fdagov-drugs-gen/documents/document/ucm073161. pdf. Accessed March 8, 2018.

12. Lester RM, Olbertz J. Early drug development: assessment of proarrhythmic risk and cardiovascular safety. Expert Rev Clin Pharmacol. 2016;9(12):1611-1618.

13. Darpo B. The thorough QT/QTc study 4 years after the implementation of the ICH E14 guidance. Br J Pharmacol. 2010;159(1):49-57.

14. International Conference on Harmonisation of Technical Requirements for Registration of Pharmaceuticals for Human Use. The clinical evaluation of QT/QTc interval prolongation and proarrhythmic potential for non-antiarrhythmic drugs E14. International Conference on Harmonisation of Technical Requirements for Registration of Pharmaceuticals for Human Use; 2005. Available from: https://www.ich.org/fileadmin/ Public_Web_Site/ICH_Products/Guidelines/Efficacy/E14/E14_Guideline.pdf. Accessed March 8, 2018.

15. Bouvy JC, Koopmanschap MA, Shah RR, Schellekens H. The costeffectiveness of drug regulation: the example of thorough QT/QTc studies. Clin Pharmacol Ther. 2012;91(2):281-288.

16. US Food and Drug Administration. Guidance for Industry ExposureResponse Relationships - Study Design, Data Analysis, and Regulatory Applications. Food and Drug Administration; 2003. Available from: https://www.fda.gov/downloads/drugs/guidancecomplianceregulatoryinformation/guidances/ucm072109.pdf. Accessed March 8, 2018.

17. Piotrovsky V. Pharmacokinetic-pharmacodynamic modeling in the data analysis and interpretation of drug-induced QT/QTc prolongation. AAPS J. 2005;7(3):E609-E624.

18. Galeazzi RL, Benet LZ, Sheiner LB. Relationship between the pharmacokinetics and pharmacodynamics of procainamide. Clin Pharmacol Ther. 1976;20(3):278-289.
19. Ferber G, Zhou M, Darpo B. Detection of QTc effects in small studies-implications for replacing the thorough QT study. Ann Noninvasive Electrocardiol. 2015;20(4):368-377.

20. Panicker GK, Karnad DR, Kadam P, Badilini F, Damle A, Kothari S. Detecting moxifloxacin-induced QTc prolongation in thorough QT and early clinical phase studies using a highly automated ECG analysis approach. Br J Pharmacol. 2016;173(8):1373-1380.

21. Grosjean P, Urien S. Moxifloxacin versus placebo modeling of the QT interval. J Pharmacokinet Pharmacodyn. 2012;39(2):205-215.

22. Shah RR, Maison-Blanche P, Duvauchelle T, Robert P, Denis E. Establishing assay sensitivity in QT studies: experience with the use of moxifloxacin in an early phase clinical pharmacology study and comparison with its effect in a thorough QT study. Eur J Clin Pharmacol. 2015;71(12):1451-1459.

23. Barbour AM, Magee M, Shaddinger B, et al. Utility of concentrationeffect modeling and simulation in a thorough QT study of losmapimod. J Clin Pharmacol. 2015;55(6):661-670.

24. Mehta R, Green M, Patel B, Wagg J. Concentration-QT analysis of the randomized, placebo- and moxifloxacin-controlled thorough QT study of umeclidinium monotherapy and umeclidinium/vilanterol combination in healthy subjects. J Pharmacokinet Pharmacodyn. 2016;43(2):153-164.

25. Florian JA, Tornoe CW, Brundage R, Parekh A, Garnett CE. Population pharmacokinetic and concentration--QTc models for moxifloxacin: pooled analysis of 20 thorough QT studies. J Clin Pharmacol. 2011;51(8):1152-1162.

26. Garnett CE, Beasley N, Bhattaram VA, et al. Concentration-QT relationships play a key role in the evaluation of proarrhythmic risk during regulatory review. J Clin Pharmacol. 2008;48(1):13-18.

27. Bloomfield D, Krishna R. Commentary on the clinical relevance of concentration/QTc relationships for new drug candidates. J Clin Pharmacol. 2008;48(1):6-8.

28. Darpo B, Garnett C, Keirns J, Stockbridge N. Implications of the IQ-CSRC prospective study: time to revise ICH E14. Drug Saf. 2015;38(9):773-780.

29. France NP, Della Pasqua O. The role of concentration-effect relationships in the assessment of QTc interval prolongation. Br J Clin Pharmacol. 2015;79(1):117-131.

30. Huh Y, Hutmacher MM. Evaluating the use of linear mixed-effect models for inference of the concentration-QTc slope estimate as a surrogate for a biological QTc model. CPT Pharmacometrics Syst Pharmacol. 2015;4(1):e00014.

31. Tornoe CW, Garnett CE, Wang Y, Florian J, Li M, Gobburu JV. Creation of a knowledge management system for QT analyses. J Clin Pharmacol. 2011;51(7):1035-1042.

32. Hossain M, Zhou M, Tiffany C, Dumont E, Darpo B. A phase I, randomized, double-blinded, placebo- and moxifloxacin-controlled, four-period crossover study to evaluate the effect of gepotidacin on cardiac conduction as assessed by 12-lead electrocardiogram in healthy volunteers. Antimicrob Agents Chemother. 2017;61(5).

33. Hong T, Han S, Lee J, et al. Pharmacokinetic-pharmacodynamic analysis to evaluate the effect of moxifloxacin on QT interval prolongation in healthy Korean male subjects. Drug Des Devel Ther. 2015;9:1233-1245.

34. Morganroth J, Wang Y, Thorn M, et al. Moxifloxacin-induced QTc interval prolongations in healthy male Japanese and Caucasian volunteers: a direct comparison in a thorough QT study. Br J Clin Pharmacol. 2015;80(3):446-459.

35. Darpo B, Benson C, Dota C, et al. Results from the IQ-CSRC prospective study support replacement of the thorough QT study by QT assessment in the early clinical phase. Clin Pharmacol Ther 2015;97(4):326-335.

36. Ferber G, Lorch U, Taubel J. The power of Phase I studies to detect clinical relevant QTc prolongation: a resampling simulation study. Biomed Res Int. 2015;2015:293564.

37. Taubel J, Wong AH, Naseem A, Ferber G, Camm AJ. Shortening of the QT interval after food can be used to demonstrate assay sensitivity in thorough QT studies. J Clin Pharmacol. 2012;52(10):1558-1565. 
38. Taubel J, Ferber G. The reproducibility of QTc changes after meal intake. J Electrocardiol. 2015;48(2):274-275.

39. Ferber G, Fernandes S, Taubel J. Estimation on the power of the food effect on QTc to show assay sensitivity. J Clin Pharmacol. 2018;58(1):81-88.

40. Taubel J, Ferber G, Lorch U, Wang D, Sust M, Camm AJ. Single doses up to $800 \mathrm{mg}$ of E-52862 do not prolong the QTc interval--a retrospective validation by pharmacokinetic-pharmacodynamic modelling of electrocardiography data utilising the effects of a meal on QTc to demonstrate ECG assay sensitivity. PLoS One. 2015;10(8):e136369.

41. Wheeler B. QTcF postural changes as positive control for TQT studies: Eliminating the moxifloxacin group. Paper presented at: DIA; June 19-23, 2011; Chicago.

42. Cardiac Safety Research Consortium. Exposure Response Analysis to Evaluate a Drug's Effect on ECG Parameter. Cardiac Safety Research Consortium; 2016. Available from: http://cardiac-safety.org/wp-content/ uploads/2016/04/1CSRC_2016_Garnett.pdf. Accessed March 8, 2018.

43. Ferber G, Zhou M, Dota C, et al. Can bias evaluation provide protection against false-negative results in QT studies without a positive control using exposure-response analysis? J Clin Pharmacol. 2017;57(1):85-95.

44. Couderc JP, Xiaojuan X, Zareba W, Moss AJ. Assessment of the stability of the individual-based correction of QT interval for heart rate. Ann Noninvasive Electrocardiol. 2005;10(1):25-34.
45. Marathe D. Regulatory perspective for using C-QTc as the primary analysis: trial design, ECG quality evaluation, evaluation of modeling/ simulation results, and decision making. Poster presented at: Cardiac Safety Research Consortium Meeting; April 6, 2016; Washington, DC.

46. Overgaard RV, Ingwersen SH, Tornoe CW. Establishing good practices for exposure-response analysis of clinical endpoints in drug development. CPT Pharmacometrics Syst Pharmacol. 2015;4(10):565-575.

47. US Food and Drug Administration. Guidance for Industry S7B Nonclinical Evaluation of the Potential for Delayed Ventricular Repolarization (QT Interval Prolongation) by Human Pharmaceuticals. Food and Drug Administration; 2005. Available from: https://www.fda.gov/downloads/Drugs/GuidanceComplianceRegulatoryInformation/Guidances/ ucm074963.pdf. Accessed March 8, 2018.

48. Nielsen J, Graff C, Hardahl T, et al. Sertindole causes distinct electrocardiographic T-wave morphology changes. Eur Neuropsychopharmacol. 2009;19(10):702-707.

49. Johannesen L, Vicente J, Mason JW, et al. Differentiating drug-induced multichannel block on the electrocardiogram: randomized study of dofetilide, quinidine, ranolazine, and verapamil. Clin Pharmacol Ther. 2014;96(5):549-558.

50. Vicente J, Johannesen L, Hosseini M, et al. Electrocardiographic biomarkers for detection of drug-induced late sodium current block. PLoS One. 2016;11(12):e0163619.
Drug, Healthcare and Patient Safety

\section{Publish your work in this journal}

Drug, Healthcare and Patient Safety is an international, peer-reviewed open access journal exploring patient safety issues in the healthcare continuum from diagnostic and screening interventions through to treatment, drug therapy and surgery. The journal is characterized by the rapid reporting of reviews, original research, clinical, epidemiological and

\section{Dovepress}

post-marketing surveillance studies, risk management, health literacy and educational programs across all areas of healthcare delivery. The manuscript management system is completely online and includes a very quick and fair peer-review system. Visit http://www.dovepress.com/ testimonials.php to read real quotes from published authors. 